\title{
The Mass Loss and Pulsation Activity in Supernova Progenitors.
}

\author{
O. A. Tsiopa \\ Central Astronomical Observatory of the Russian \\ Academy of Sciences, Pulkovo 196140, St.Petersburg, Russia
}

Abstract. The wind-formed regular structures revealed in early super-
nova (SN) spectra can be a result of the radial pulsation of the precursor.

\section{Introduction}

The near maximum light SN spectra contain the features, formed by the wind ejected in the last few months prior to the explosion.

Dopita et al. (1984) discovered a so called "superwind", which had been ejected with the extremly high velocities of $3000 \mathrm{~km} / \mathrm{s}$ immediately prior to the SN event. The fascinating change of the velocity shifts in type II SNe $1983 \mathrm{~K}$ (Niemela et al. 1985) and type Ia 1990M (Polcaro \& Viotti 1991) are evidence of the existence of complicated, but rather regular structures in the wind at the scales of less than $10^{16} \mathrm{~cm}$. Recently (Tsiopa 1995) the observational facts were united in a common dynamical model.

\section{The winds of the SN precursors Type II and Ia}

Though the SN envelope velocities of SNe type II are less, than in SNe type I, their winds are extremely fast (up to $2000-4000 \mathrm{~km} / \mathrm{s}$ ). The wind being highly variable and dense, the pronounced colliding wind-formed structures occur close to the SN progenitor. The system of interacting wind layers with different deviation of symmetry and increasing velocities can form the distinct prolate ellipsoidal shell around the SN progenitor just before the explosion.

The presupernova winds of SN type I are very difficult to detect: they are less massive, slower (hundreds $\mathrm{km} / \mathrm{s}$ ) and almost immediately swept up by the SN envelope expanding with very high speed. For the case of $1990 \mathrm{M}$ the model implies the existence of a shell formed by the matter with a maximum velocity of $1200 \mathrm{~km} / \mathrm{s}$ and a minimum velocity of $600 \mathrm{~km} / \mathrm{s}$.

Judging from the SN envelope expansion velocity the wind-formed structures under consideration are situated at the radii from the explosion centre of $10^{14}-10^{15} \mathrm{~cm}$ for SN $1983 \mathrm{~K}$ and $3-6 \times 10^{15} \mathrm{~cm}$ for SN $1990 \mathrm{M}$. The time of shell ejection can be estimated as $6 \times 10^{6} \mathrm{~s}$ for SN 1983K and $5 \times 10^{7} \mathrm{~s}$ for SN $1990 \mathrm{M}$. Such rather small distances and short periods of the regular structure formation imply the presence of a high initial gradient of density and velocity in the wind matter. 


\section{Discussion}

An extraordinary activity of the precursor can be suspected even for a single star. During the periods of interior reconstruction (preceding the SN type II event) even the outer parts of the star are likely to be in the unstable state. Strong pulsation with different modes can be evoked. Such processes as severalmode resonant coupling are probable to determine the mass-loss rates in critical periods of stellar evolution. During expansion the star throws away the very outer part of its envelope (or, in other words, the stellar wind increases dramatically) and an expanding shell is formed. In this case the precursor would be surrounded by a system of interacting shells (Tsiopa 1990)

It should be noted that the majority of the pulsating stars are located in the same region of the Hertzsprung-Russell diagram as expected for type II SN precursors. And though the modern SN theory does not provide any indications of the resonance nature of the explosion (deflagration regimes of the thermonuclear explosion of a degenerate core can provide only very short period pulsation (10 seconds), which cannot correlate with the envelope motions), there are some possible additional energy sources which can function in phase correlation with proper pulsation of the star at the last stages of its evolution. First of all, it can be the flaring up of the nuclear shell source, then the process of the neutronization can split into stages, and at last for non-Fe core (O-C, for example) the outer parts of the core can also flare up. It is possible that the explosion itself is encouraged by the resonance pulsation.

As for the SN type Ia precursors some other mechanisms are certainly to be considered. However the close systems buried in a common envelope (or one compact object inside the extended atmosphere of the other component) is in some sense indistinguishable from the rotating pulsating star. The forming circumstellar environs may occur in a similar way. Perhaps, the eccentricity of the orbit in binary system can be treated as a trigger in generating instabilities and resonance effects.

The recurrent ejections of matter with different velocities can result in formation of axial colliding wind formed structures. A very energetic individual pulsation of supernova precursor just before the explosion event produces a shell, perhaps, discovered in the early spectra of SN 1984E.

\section{References}

Dopita, A.M., Evans, R., Cohen, M., \& Schwarts, R.D. 1984, ApJ, 287, L69

Niemela, V.S., Ruiz, M.T., \& Phillips, M.M. 1985, ApJ, 289, 52

Polcaro, V.F., \& Viotti, R. 1991, A\&A, 242, L9

Tsiopa, O.A. 1990, Astronomicheskj Tsirkular, No. 1554, 23

Tsiopa, O.A. 1995, in: K.A. van der Hucht and P.M. Williams (eds.), Wolf-Rayet Stars: Binaries, Colliding Winds, Evolution (Dordrecht:Kluwer), 425 